\title{
Laser Source for Wireless Power Transmission in Space
}

\author{
Victor V. Apollonov \\ Prokhorov General Physics Institute RAS, Moscow, Russia \\ Email: vapollo@kapella.gpi.ru
}

Received 20 September 2015; accepted 8 October 2015; published 14 October 2015

Copyright (C) 2015 by author and OALib.

This work is licensed under the Creative Commons Attribution International License (CC BY). http://creativecommons.org/licenses/by/4.0/

(c) (i) Open Access

\begin{abstract}
For many years the attempts to create a super long conductive channel were taken in order to study the upper atmosphere and to settle special tasks, related to the energy transmission. There upon the program of "Impulsar" creation represents a great interest, as this program in a combination with high-voltage high repetition rate electrical source can be useful to solve the above mentioned problems. It looks like as some kind of "renaissance of $\mathrm{N}$. Tesla ideas" for the days of high power lasers. In the experiments the $\mathrm{GDL} \mathrm{CO}_{2}$-laser and solid state Nd YAG laser systems had been used. Theoretical and experimental investigation of high repetition rate P-P mode of operation for high power lasers (COIL, HF/DF) had been provided. The experimental realization of P-P regime has been demonstrated for $100 \mathrm{KW}$ GDL and $1 \mathrm{~kW}$ LD pumped SSL. More than two orders of magnitude ratio of peak to average power with minimal energy loss have been obtained. High efficiency and excellent beam quality of disk laser mean that it can be widely used in modern science and industry. Disk laser idea, suggested by N. G. Basov 52 years ago, after implementation in the mono-module disk geometry can be used effectively for many important challenges faced by science and technological advances of the future. Today we can say that creation of megawatt class mono-module P-P disk laser with a large cross section of the active medium opens up great prospects for its use for solving of the set of problems: small satellites launching by lasers, formation of super-long conducting channels in space (vacuum and atmosphere), cleaning of the near-Earth space from the debris and so on.
\end{abstract}

\section{Keywords}

Laser, Conductive Channel, Laser Spark, Electric Discharge, Pulse-Periodic Laser, Energy Transmission

Subject Area: Applied Physics

\section{Introduction}

At present time, many laboratories of the world still continue the search for an efficient laser-based lightning 
protection system [1]-[3] and for producing a controlled high-conductivity channel in the atmosphere [4]-[8], capable of conducting a short-circuit current [9] in a natural or artificial electrical circuit. Laser-based lightning protection systems, as is known, rely on the so-called long laser spark, which provides the conditions for connecting a thunderstorm cloud with a grounded metal rod, i.e., a classical lightning rod. Maximum lengths $(\sim 16$ $\mathrm{m}$ ) of the laser-spark-controlled electric discharge at a voltage of $3000 \mathrm{kV}$ were obtained by our team in Russia and Japan [3] using a $0.5-\mathrm{kJ}$ pulsed $\mathrm{CO}_{2}$ laser with spherical optics. Such control conductivity channels can be used in energy transmission, overvoltage protection systems, transport of charged particle beams, plasma antennas, etc. [8].

Our work demonstrated the feasibility of converting high-power wide-aperture laser radiation to the pulseperiodic (P-P) lasing mode by way of self-injection of the modulated fraction of output radiation without a sacrifice in output radiation power in comparison with the stationary lasing mode. In this case, the peak output power can exceed the average output power by more than two orders of magnitude. For P-P modulation with a pulse length of $50-100 \mathrm{~ns}$ the peak power is much greater than $100 \mathrm{~kW}$ for $1 \mathrm{~kW}$ average power $(\mathrm{CW})$ laser. Applicability of the method and variations of temporal structure of radiation for other type of high power lasers (HF/DF; COIL) have demonstrated the possibility of Q-switched mode realization with much similarity to GDL shape of radiation, given experimentally.

Another important question arises as to how the average power above the level of one megawatt can be achieved on the basis of solid-state laser as the most efficient one. And this power is needed to address many problems associated with the removal of debris from near-Earth space, with launching of missiles with the help of lasers, and with the creation of long-range conducting channels for wireless power transmission in space and others. Fiber lasers are not applicable for these purposes because of the smallness of the area of the exit pupil of the fibers and hence the impossibility of operation of such lasers in the high-frequency P-P regime with high peak power at an average power of a few hundred of kilowatts. The laser system based on "slabs" also seems hard to implement as adjustment of the system and its maintenance in a safe operation mode is comparable to the complexity of working with a multi-element system for the solution to the problem of controlled thermonuclear fusion. Proceeding from the above, the answer is quite clear: such a laser can and should be based on the mono-module disk laser geometry! High repetition rate P-P mode of operation should be compatible with this type of the laser as well.

\section{Basic Results in the Past}

It was shown in [10] [11] that a laser spark produced using conical optics demonstrates very attractive characteristics from the point of view of formation of conductivity channels in the atmosphere. Currently, among the huge variety of different lasers, only two types are being actively studied to be used in the formation of laser-spark-controlled high conductivity channels in the atmosphere: submicrosecond pulsed gas and chemical lasers $\left(\mathrm{CO}_{2}, \mathrm{DF}\right)$ and femtosecond solid-state lasers [4] [6] [12]. The main advantage of such a laser is the ability of producing ionized channels (filaments) with a characteristic diameter of about $100 \mu \mathrm{m}$ in atmosphere along the laser beam propagation direction. With an estimated electron density of $10^{16} \mathrm{~cm}^{-3}$ in these filaments and the laser wavelength in the range of $0.5-1.0 \mu \mathrm{m}$, the plasma hardly absorbs the laser light. In this case, the length of the track consisting of many filaments is determined by the laser intensity and can reach many miles at a femtosecond pulse energy of $\sim 100 \mathrm{~mJ}$. However, these lasers could not be used to form long high-conductivity channels in atmosphere. The ohmic resistance of the thus formed conducting channels turned out to be very high, and the gas in the channels could not be strongly heated (the femtosecond laser energy is less than $\leq 1 \mathrm{~J}$ ). In particular, an electric breakdown (EB) controlled by radiation of a femtosecond solid-state laser was implemented in [5] [6] only at a length of $3 \mathrm{~m}$ (with a voltage of $2 \mathrm{MV}$ across the discharge gap).

As was shown in [13] [14], to form a high-conductivity channel in the atmosphere, which could commute an artificial or natural discharge (lightning), the gas must be heated to a temperature from 6000 to $8000{ }^{\circ} \mathrm{K}$, when thermal ionization begins. This condition is crucial, because the high initial electron density in the atmosphere is a necessary but not sufficient condition for realizing high conductivity in a long atmospheric channel. As was mentioned in [13], it is important not only to produce but also to maintain the electron density in an atmospheric channel that is sufficient for the breakdown. During gas heating thermal ionization becomes the main mechanism of electron 'production' in the channel. Specifically this is the main reason why the streamer mechanism cannot lead directly to an electric breakdown in the presence of high electron density (filaments) in a cold channel [13]. 
In the case of submicrosecond $\mathrm{CO}_{2}$ or DF lasers with a high energy per pulse (several hundreds of joules), long conducting laser-plasma channels can be formed with conical optics. In contrast to spherical optics, one can use methods of lens dynamic correction to increase aberrations and elongate the focal segment by passing to a conical phase front [11]. For example, an axicon transforms the phase front of a plane wave so that it becomes conical rather than spherical and converges to the $\mathrm{z}$ axis at an angle $\gamma$. For small angles $\left(\gamma<<1^{\circ}\right)$, it is simply expressed in terms of the angle $\gamma$ at the axicon base and the refractive index $N$ of the axicon material: $\gamma=(\mathrm{N}-1) \alpha$. Each ring element of a conical wave front with a radius $\mathrm{R}$ and width $\delta \mathrm{R}$ is focused into an axial element of length $\delta \mathrm{z}=\delta \mathrm{R} / \gamma$ and the entire focal segment has the length $\mathrm{L}=\mathrm{R} / \operatorname{tg} \gamma=\mathrm{R} / \gamma$ [11]. This length can be made significant. For example, at $\mathrm{R}=10 \mathrm{~cm}$ and $\gamma=0.5^{\circ}$, it amounts to $\mathrm{L} \sim 11 \mathrm{~m}$. The cylindrical symmetry of focusing suggests that the type of transverse intensity distribution is independent of the coordinate $\mathrm{z}$, a situation corresponding to a "diffractionless" wave beam. In reality, such beams also undergo diffraction; however, the diffraction energy loss from the central part of the beam is compensated for by the distributed lateral supply of radiation. Note that the theoretical length of the focal segment formed by the axicon is independent of the laser beam energy and power and is determined by only the beam diameter. At $\mathrm{R}=100 \mathrm{~cm}$ and $\gamma=0.5^{\circ}$, it amounts to $\mathrm{L} \sim$ $110 \mathrm{~m}$. According to our estimates, when using $\mathrm{CO}_{2}$ lasers with an energy of $\sim 5 \mathrm{~kJ}$ per pulse, the length of these channels for wide laser beams and conical optics can be fairly large (much more than $100 \mathrm{~m}$ ).

When laser radiation is focused by an axicon in a real experiment, the longitudinal intensity distribution I(z) along the focal length $L$ depends on $z$, since the transverse intensity distribution $I(R)$ of the input beam is transformed by the axicon into the longitudinal distribution I $(\mathrm{z})$ of the Bessel beam. When the radiation intensity in the beam reaches the threshold, there occurs a breakdown in the medium and an extended plasma channel is formed along the focal length of the axicon [11]. Initially, the channel has a diameter of $20-100 \mu \mathrm{m}$ and a length that is on order of the focal length $\mathrm{L}$ of the axicon. If the breakdown is stable, the plasma channel, in contrast to the case of spherical optics, becomes continuous for few nanoseconds. The density of the plasma, depending on the parameters of the medium, the wavelength $\lambda$, and other experimental conditions, varies from $10^{17}$ to $3 \mathrm{~cm}^{-3}$, the temperature reaches $30-40 \mathrm{eV}$, and specific conductivity is $10(\mathrm{Ohm} \cdot \mathrm{m})^{-1}$. A few microseconds later the channel expands to $10 \mathrm{~mm}$. Accordingly, the temperature drops to $1 \mathrm{eV}$ and retains at this level for about $100 \mu \mathrm{s}$, followed by a relaxation of the plasma [11]. There are no discontinuities in the case of axicon based sparks that are characteristic of sparks produced by focusing radiation by spherical optics. A similar pattern was observed at laser energies below $300 \mathrm{~J}$ [8]. In the photograph of a CLS fragment in Figure 1(a) and Figure 1(b) the longitudinal structure of the spark looks like a set of bright tapered elements, which are in contact and equally spaced. Figure 1(c) shows a photograph of the electric breakdown in the atmosphere for a channel formed by a CLS. When studying the spark between two rods immersed in plasma and spaced by $\mathrm{d}=80$ $\mathrm{cm}$ [8], an electric breakdown occurred at a laser energy of $140 \mathrm{~J}$ and minimum average electric field strength $\mathrm{E}_{\min } \approx 77 \mathrm{~V} / \mathrm{cm}$.

The up-to-date experiments aimed at implementing laser-spark-guided electric discharges shows another fundamental difference in the case of femtosecond solid-state and long-wavelength lasers. In the presence of a laser-plasma channel composed of filaments, the breakdown voltage decreased by $30 \%$ [15]. The use of $\mathrm{CO}_{2}$ and DF lasers made it possible to reduce it by a factor of more than 10 .

As was pointed out in [16], the guiding properties of CLS (with a high voltage delayed by $10-20 \mu \mathrm{s}$ ) are similar to the action of a chain of metal balls. After an optimal time of $\sim 10-20 \mu \mathrm{s}$, the expansion of individual breakdown 'zones' leads to the formation of a continuous quasi-cylindrical conducting channel with a low gas density ( $\sim 0.1$ of the normal density), high equilibrium temperature $(2700-2900 \mathrm{~K})$, and electron density in the range from $10^{7}$ to $10^{8} \mathrm{~cm}^{-3}$.

Another approach to the formation of a conducting channel relies on the application of a P-P laser. It was shown in [17] that electric-discharge and gas-dynamic lasers with a high peak power can operate in a high-frequency P-P regime. This lasing regime allows one to produce a long conducting channel in the atmosphere, which exists for a long time. To this end, it is necessary to align a laser beam with an inverted Cassegrain telescope system, which consists of two spherical mirrors. The first (main) mirror of large diameter is immobile, while the second mirror can move according to a certain law. When moving the second mirror, the common real focus of the system changes its position in time from infinity to some minimum value, which is determined by the optical scheme of the telescope. Calculations show that, at a certain repetition frequency of laser pulses and a certain motion law for the second mirror, continuous extended plasma channels can be formed. However, practical implementation of this scheme with a "running" focus meets a number of fundamental difficulties. The 


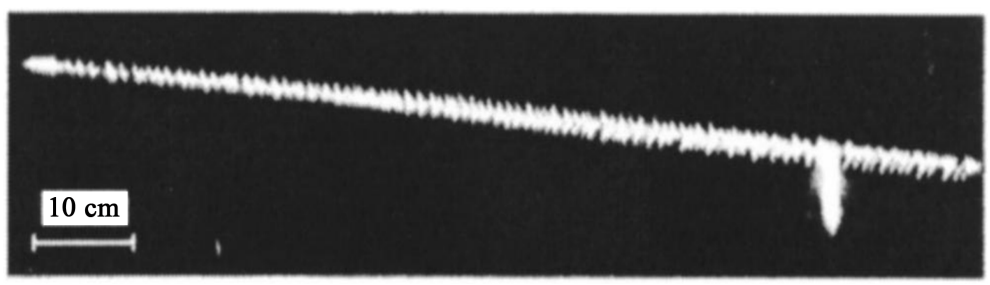

(a)

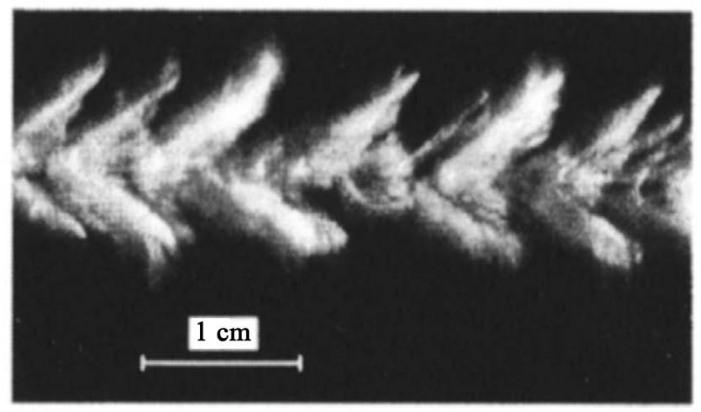

(b)

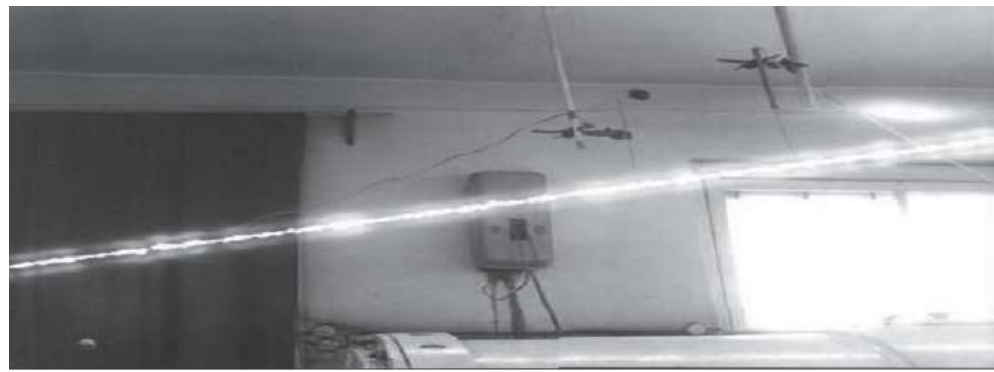

(c)

Figure 1. (a) and (b) The longitudinal structure of the spark in air; (c) Photo of the electric breakdown in the atmosphere for a channel formed by a CLS.

light distribution in the focal plane, yielded by a well correlated lens, is in essence due to the Fraunhofer diffraction. The specific features of the out-of-focus 3D monochromatic images of a point source, obtained with a round hole, were considered for the first time by Lommel. Along the optical $\mathrm{z}$ axis, the length of the focal segment that determines the longitudinal size of the energy-accumulation region depends quadratically on the focal length of the system. The transverse size of this region is proportional to the focal length and the angular divergence of laser radiation; therefore, the volume within which energy is concentrated is proportional to the focal length in the fourth power. For this reason, to form a homogeneous plasma channel several kilometers long, one should use a P-P laser with an accurately shaped pulse energy and the small telescope mirror moving according to a complex program. A practical implementation of this method with a $\mathrm{P}-\mathrm{P} \mathrm{CO}_{2}$ laser with an average power of $1 \mathrm{~kW}$ yielded a continuous plasma channel $\sim 1 \mathrm{~m}$ long [18].

\section{Conducting Channel Formation}

A completely different approach to the formation of a conducting channel can be realized using a high repetition rate P-P lasers [19]. Motion of a light driven engine under irradiation by a high-frequency P-P laser with a pulse energy sufficient for producing a breakdown in the focus of the optical system of a laser reactive engine (LRE) gives rise to a continuous conducting channel in air as a result of the formation of a mixture of aerosol [20] with atmospheric air and its subsequent ionization [19]. In this case, according to the calculations, the aforementioned range of laser pulse repetition rates provides continuity of the conducting channel at optimal velocities of the focusing system. Indeed, each laser pulse focused by the optical system forms some extended plasma region with a high conductivity, which occupies a relatively small segment of the focusing system trajectory. If the la- 
ser pulses have a low repetition rate [21], these regions will form somewhat like a dashed line at some velocities of the focusing system. If the pulse repetition rate exceeds $10 \mathrm{kHz}$ and the motion velocities are optimal for solving the problem stated, these high-conductivity regions will have no discontinuities, and a continuous conducting channel will be formed. However, when the moving optical system enters rarefied atmospheric layers, a problem of medium deficit arises. To solve it, the displaced optical system must have an efficient source of an appropriate medium (a material containing microscopic metal particles, which can easily be sublimated under laser irradiation) in the vicinity of the focus. In particular, one can use copper oxide (CuO) nano-powder [22], which provides synthesis of conducting aerosol copper particles upon laser heating. The presence of aerosol particles of heavy metals in the sublimated material makes it possible to increase the specific impulse of the LRE thrust, simultaneously with increasing the channel conductivity.

To implement a long conducting channel, one must solve several problems:

1) Development of a high-frequency P-P laser with an average power more than $100 \mathrm{~kW}$ and a peak power sufficient for initiating a breakdown in the focus of the LRE optical system;

2) Development of a system of dynamic correction for the wave front of a wide-aperture laser beam with a diameter $\mathrm{D}>10 \mathrm{~cm}$ in the presence of small-scale turbulence in atmosphere;

3) Consideration of the laser radiation absorption and scattering by aerosol particles in the LRE exhaust;

4) Alignment of the starting position of LRE with the laser and the pulsed high-voltage (up to several MV) source;

5) Obtainment of maximally possible specific impulse of the LRE thrust to reduce the time of aerosol channel formation;

6) Choice of the material of aerosol heavy-metal particles and their dispersion composition that could provide high channel conductivity upon heating to the sublimation temperature in the LRE;

7) Determination of the lower limit for the size of dispersion aerosol particles to ignite electric breakdown of a channel capable of transmitting a current, corresponding to a short-circuit current in natural or artificial electric circuits at a minimally possible average strength of electric field.

We consider as a very important the problems of determining the dynamics of change in the channel conductivity at long delay times and its maintenance at the optimal level by introducing additional high-frequency generators into a chain.

\section{High-Frequency Pulse-Periodic Solid-State and $\mathrm{CO}_{2}$ Gas-Dynamic Lasers}

Currently, in technological processes and physical experiments with high average power (over $1 \mathrm{~kW}$ ) lasers, advantage is taken only of sources operating in two regimes - CW and P-P with a repetition rate from a few to hundreds of $\mathrm{Hz}$ at pulse durations within tens to thousands of microseconds and even milliseconds. High-frequency P-P laser systems with a high average power, which provide the required pulse train durations from a few to hundreds of nanoseconds, allows implementation of a fundamentally different mechanism of interaction of radiation with matter-sublimation (ablation) ensuring a local energy release not only in space but also in time [23] [24]. This results in an explosive local evaporation of a substance without an intermediate liquid phase. This mechanism can significantly extend the range of physical and technological applications of laser sources.

High-frequency intra-cavity loss modulation approach as most effective way of high repetition rate P-P lasers creation was realized for a solid-state laser with flash lamp and LD pumping for the level of average output power up to $1 \mathrm{~kW}$. Such lasers can be fabricated by using the existing experience in the field of calculation and design of laser resonators, which provide their operation at the stability boundary with simultaneous optical decoupling of intra-cavity elements. In the case of industrial applications the stability of output can be reached with the help of an active rear mirror, which provides stabilization of the fundamental mode in the cavity. The experimental setup uses the active cavity, which includes two active mirrors-a rear mirror with a variable radius of curvature and an output mirror-with variable reflectivity varying along the radial coordinate. Using the system of active mirrors has made it possible to significantly improve laser radiation quality. The parameter $\mathrm{M}^{2}<$ 10 for the case of lamp pumped laser was obtained with decreasing of power down to $350 \mathrm{~W}$. Therefore, the increase in the power density in the focal plane of the lens exceeded one order of magnitude. Besides, improving the divergence of the output radiation creates prerequisites for the normal operation of standard acousto-optic modulators used in single-mode lasers. Thus, the Q-switched regime can be realized in the frequency range from 10 to $30 \mathrm{kHz}$ at an output power of $250 \mathrm{~W}$. When using a similar approach for the case of LD pumped laser, the P-P regime at an output power up to $1 \mathrm{~kW}$, with a slight deterioration in quality of the output radiation, has been 
implemented (Figure 2). Very important to emphasize the very little lose of energy during the transformation process of $\mathrm{CW}$ into P-P regime (Figure 3).

Low-average-power high repetition rate P-P solid-state laser can be found at the market, but high-frequency P-P $\mathrm{CO}_{2}$ lasers with the same or bigger output power that is suitable for many purposes are absent at all. However, the use of high-frequency P-P $\mathrm{CO}_{2}$ lasers will fundamentally expand the scope of their applications. The possibility of high-frequency P-P operation of lasers of virtually any level of power has been demonstrated by us both theoretically and experimentally. Experimental testing under laboratory conditions suggests the principle possibility of transferring the available technology to industry. The basic requirement for industrial lasers is the high reliability and long-term trouble-free operation. In this connection, it is desirable to ensure the low serviceability of equipment. In this regard, the development of industrial laser sources requires additional applied research to obtain maximum stability of the output characteristics, reliability and service life of the laser. The main emphasis of our work was directed to the optimization of optical schemes of laser resonators operating in the high-frequency P-P regime and search for solutions that provide high radiation resistance of optical elements of lasers [25].

The high-frequency P-P regime fundamentally differs from $\mathrm{CW}$ and low-frequency operation modes by the mechanisms of interaction of laser radiation with matter. Experiments show that in the case of high-frequency P-P laser sources, it is needed to significantly increase the ranges of variation of many interaction parameters.

The above objectives can be achieved through physical foundations, elaborated by our team in this area since 1993 [26]. The emphasis is on the two main approaches to implementation of the high-frequency P-P regime in the case of high power laser. In the lasers with a high average output power (GDL, HF/DF, COIL, Mono-module disk laser (MMDL) use is commonly made of unstable resonator configurations owing to the large cross sec-

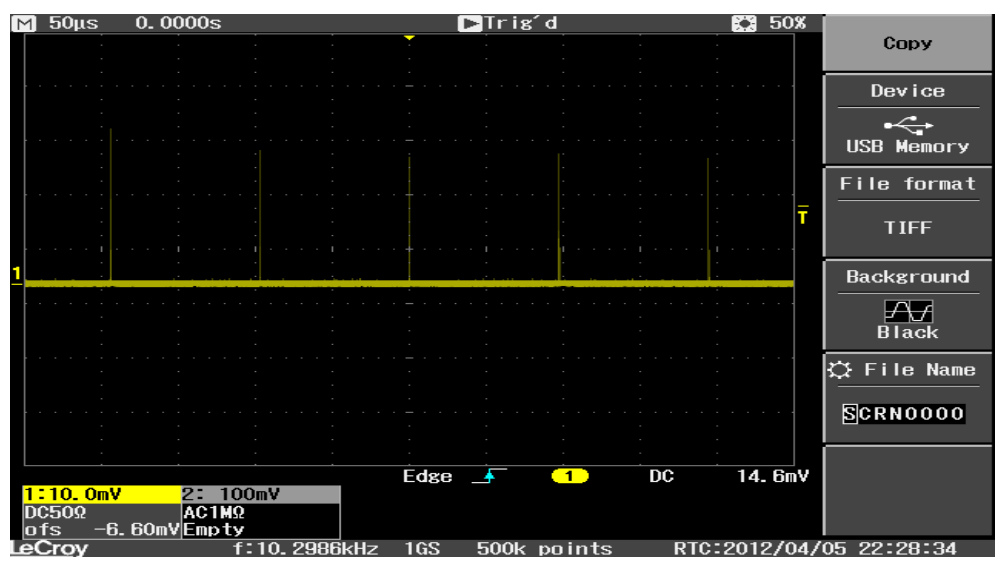

Figure 2. Continuous train of pulses for Nd: YAG laser, $\mathrm{P}=1 \mathrm{~kW}$.

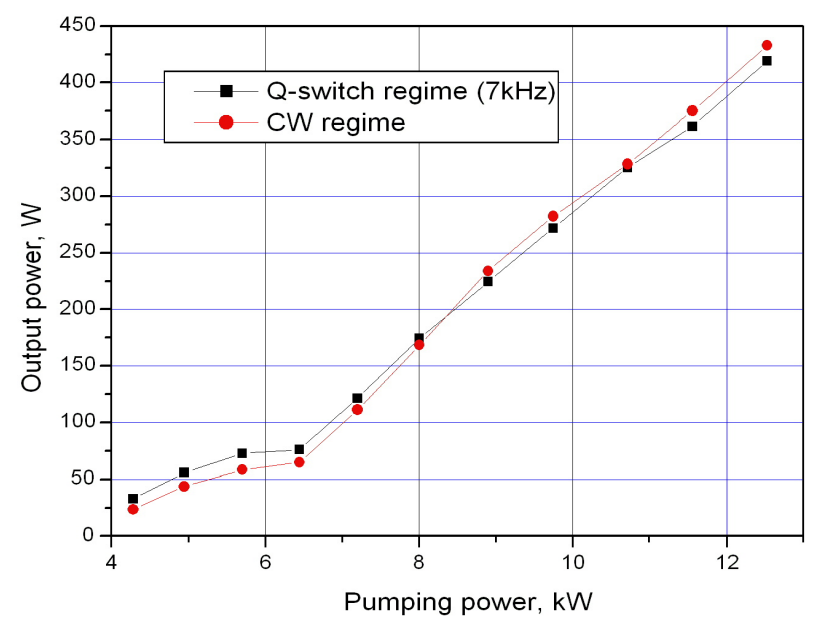

Figure 3. Output power for CW and P-P modes of operation. 
tion of the active medium. In resonators of this type, externally injected low-power beams may exert a significant effect on the characteristics of output radiation. One way to realize the control regime is the self-injection of radiation extraction from the resonator and return of a part of radiation after changing its spatial-temporal characteristics. The transition to the transient lasing mode is effected through the modulation of the self-injecting beam. Earlier, a study was made of laser versions with radiation self-injection into the paraxial resonator region. However, analysis showed that the power of the beam injected into the paraxial beam region should be not too many orders of magnitude less than the output laser power necessary for efficient control of the resonator of a continuously pumped laser, unlike the pulsed laser systems with regenerative amplification, where the signal difference is significant.

The self-injection of a part of output radiation through the resonator periphery is more efficient: on return to the paraxial resonator region, the injection power significantly rises due to the large number of passages to play the dominant role in the formation of output radiation. In the case of a traditional resonator, the role of waves converging to the resonator axis was found to be insignificant, because their source is a narrow region with a small relative area at edge of the output mirror; accordingly, the power of the control wave injected into the resonator is low. This wave has a large divergence, and only its small part (of the order of $1 / \mathrm{Nf}$, where $\mathrm{Nf}>>1$ is the Fresnel number) participates in lasing.

The effect of injection wave on the resonator characteristics can be enhanced by matching the beam phase with the resonator configuration and increasing the radiation power returned. In this case, the propagation direction and the wave front curvature of the injection beam should be so matched with the resonator configuration that the injection beam concentrates, after a relatively large number of passages through the resonator, near the optical resonator axis and transforms to a divergent wave that forms the output radiation. The injection beam energy should be high enough to exceed, after its arrival to the resonator axis, the saturation energy of the active medium.

The experimental configuration was realized in a gas-dynamic $\mathrm{CO}_{2} \mathrm{P}=100 \mathrm{~kW}$ laser with the following parameters: the length of the active medium $\mathrm{L}_{\mathrm{a}}=1.2 \mathrm{~m}$, the unsaturated gain coefficient $\mathrm{g}_{0}=0.6 \mathrm{~m}^{-1}$, the time it takes the active medium to transit the resonator $\tau=0.92 \times 10^{-4} \mathrm{~s}$, the relaxation time $\tau_{\mathrm{p}}=2.76 \times 10^{-4} \mathrm{~s}$, the total go-round resonator time $\tau_{\mathrm{f}}=4.2 \times 10^{-9} \mathrm{~s}$, the luminescence lifetime $\tau_{\mathrm{l}}=5 \mathrm{~s}$, the resonator magnification factor M $=1.45$, the diameter of output laser aperture $\mathrm{a}=0.08 \mathrm{~m}$.

The laser resonator is made up of two spherical mirrors with rectangular apertures, which provided a geometrical amplification factor of 1.45. The active medium travels across the optical resonator axis. In what follows all theoretical and experimental data are given for a laser with the above parameters.

A part of the output laser radiation was diverted by an inclined metallic mirror to the injection beam formation system consisting of two spherical mirrors with conjugate focal planes. In the vicinity of the focal plane there formed the waist of the branched part of the laser beam, and a modulator was placed near the waist. The modulator location was so selected that the laser beam completely filled the aperture of the modulator. The maximal modulation frequency in our experiments was equal to $33 \mathrm{kHz}$.

The duration of an individual pulse was about 100 - $150 \mathrm{~ns}$. We emphasize that the recorded pulse duration was limited by the measuring path bandwidth equal, as noted above, to $50 \mathrm{MHz}$. The amplitudes of individual pulses exceeded the average value of output power by factor-100. The average output power was measured with a calorimeter cooled by running water. It is noteworthy that the average output power in the P-P mode was equal to the output power in the CW laser-operating mode (Figure 3). Good agreement between the experimental and theoretical data for frequencies ranging up to $30 \mathrm{kHz}$ testifies to the adequacy of the proposed model and the possibility of employing this method at higher frequencies to convert a CW laser radiation to the P-P operating mode [27].

\section{Pulse-Periodic HF/DF and COIL Lasers}

We have performed the set of calculations for two the most powerful lasers of our days with the goal to get possible temporal structure of radiation and peak power value of pulses after $\mathrm{CW}$ mode transformation into P-P mode. The basic parameters for calculations are presented in Table 1 for HF/DF laser and Table 2 for COIL.

\section{a) HF/DF laser}

For the experimental configuration one can conclude:

P-P mode is valid for the modulation frequency $60-600 \mathrm{KHz}$. The depth of modulation will be chaotic and structure-bundles of pulses for the frequency gap: $100-250 \mathrm{KHz}$. The duration of bundles will be controlled by 
Table 1. Parameters of HF/DF laser.

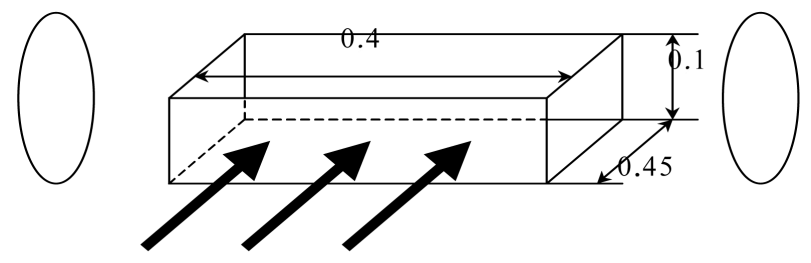

$\mathrm{V}=2500 \mathrm{~m} / \mathrm{s}$

\begin{tabular}{cccc}
\hline Parameter & Value & Parameter & Value \\
\hline $\mathrm{La}$ & $0.4 \mathrm{~m}$ & $\tau$ & $0.18 \times 10^{-4} \mathrm{~s}$ \\
$\mathrm{Lr}$ & $1.0 \mathrm{~m}$ & $\tau_{\mathrm{p}}$ & $(2-3) \times 10^{-5} \mathrm{~s}$ \\
$\mathrm{~g}_{0}$ & $5-10 \mathrm{~m}^{-1}$ & $\mathrm{M}$ & 1.4 \\
$\mathrm{C}$ & $3 \times 10^{8} \mathrm{~m} / \mathrm{s}$ & $\tau_{\mathrm{f}}$ & $3.3 \times 10^{-9} \mathrm{~s}$ \\
$\mathrm{~A}$ & $0.08 \mathrm{~m}$ & $\tau_{\mathrm{l}}$ & $20 / 330 \mathrm{~s}$ \\
\hline
\end{tabular}

where: La—active media length; $\mathrm{g}_{0}$ —unsaturated gain coefficient.; g—saturated gain coefficient.; $\mathrm{c}$-light velocity; $\tau$-time of active media travel across the resonator; $\tau_{\mathrm{p}}$-relaxation time; $\tau_{\mathrm{f}}$ round trip time; $\tau_{\mathrm{l}}$-luminescence time; $\mathrm{M}$-magnification of resonator; a-diameter of aperture.

\section{Table 2. Parameters of COIL.}
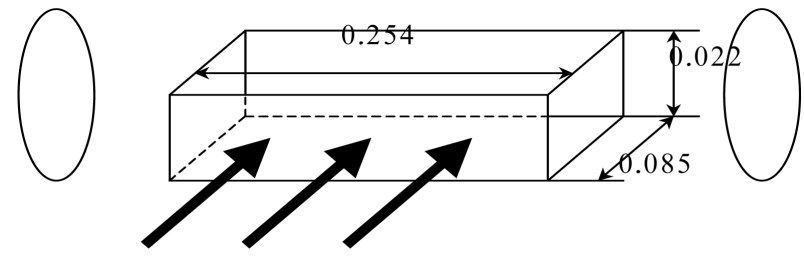

$\mathrm{V}=1200 \mathrm{~m} / \mathrm{s}$

\begin{tabular}{cccc}
\hline Parameter & Value & Parameter & Value \\
\hline $\mathrm{La}$ & $0.254 \mathrm{~m}$ & $\tau$ & $0.71 \times 10^{-4} \mathrm{~s}$ \\
$\mathrm{Lr}$ & $1.5 \mathrm{~m}$ & $\tau_{\mathrm{p}}$ & $(10-12) \times 10^{-5} \mathrm{~s}$ \\
$\mathrm{~g}_{0}$ & $56.9 \mathrm{~m}^{-1}$ & $\mathrm{M}$ & 1.2 \\
$\mathrm{C}$ & $3 \times 10^{8} \mathrm{~m} / \mathrm{s}$ & $\tau_{\mathrm{f}}$ & $5 \times 10^{-9} \mathrm{~s}$ \\
$\mathrm{a}$ & $0.04 \mathrm{~m}$ & $\tau_{\mathrm{l}}$ & $2 \mathrm{~s}$ \\
\hline
\end{tabular}

filling factor. For the frequencies more than $250 \mathrm{KHz}$ the depth of modulation will reach $100 \%$. (Ruff pulses structure is very similar to the structure of transverse modes biting). For frequencies more than $300 \mathrm{KHz}$ one can get a classical regime of regular pulsed train. Duration of pulses should be in the gap: $100-150$ ns. The ratio P peak/P aver. is in the gap $100-1000$.

b) COIL

All parameters of the system have the same definitions as for the table mentioned in the beginning of the paragraph. From the data above one can conclude: P-P mode should start for the modulation frequency more than $20 \mathrm{KHz}$. The depth of modulation will be chaotic and structure-bundles of pulses. The duration of bundles will be controlled by filling factor. For frequency more than $100 \mathrm{kHz}$ the depth of modulation will reach $100 \%$.For frequencies more than $150 \mathrm{kHz}$ - classical regime of regular pulses train. Duration of pulses should be less than $150 \mathrm{~ns}$. The ratio $\mathrm{P}$ peak/P aver. is in the gap 100 - 1000.

\section{Bright Future-High Power Mono-Module Disk Laser}

The scaling laws for the MMDL design show that the power limit for $\mathrm{CW}$ operation is far beyond $100 \mathrm{~kW}$ for a 
single disk and the pulsed energy limit is higher than $100 \mathrm{~J}$ from one disk in pulsed operation [27]. By surface cooling of the disk, the optical distortion of the laser beam is low and therefore operation of the thin disk laser is possible in fundamental mode at extremely high output power. The disk laser concept is a laser design for diode-pumped solid-state lasers, which allows the realization of lasers with high output power, having very good efficiency and also an excellent beam quality. Since the first mentioning of the principle suggested in 1962 and demonstrated in 1964 by acad. N.G. Basov with colleagues [28] the output power of disk laser has been increased to $4 \mathrm{~kW}$ in CW-operation. Disk lasers (chain of disks) with up to $30 \mathrm{~kW}$ are now commercially available for materials processing [29]-[31]. The beam quality of all commercially available disk lasers is always better than for rod lasers of similar power level, lasers with up to $100 \mathrm{~W}$ of power are available with fundamental mode (M2 < 1.2). Additionally, the electrical efficiency is higher than that of all other commercially available solid-state lasers with similar level of power. The disk laser design also allows highly efficient pulsed operation as a high repetition rate P-P laser or as a laser amplifier. In particular, the generation and amplification of ultra-short pulses is possible with a very high average power and also high efficiency. These properties of disk lasers will open the way to a completely new class of pulsed laser systems for materials processing. With all its outstanding features, the disk laser will not only replace classical laser systems in many applications but in particular it will create new markets for laser technology. One of the outstanding features of the disk laser is its excellent beam quality, which results from the surface cooling of the laser disk. The laser crystal is shaped as a disk with a diameter not more than $10 \mathrm{~mm}$, depending on the output power/energy and a thickness of up to 500 $\mu \mathrm{m}$, depending on the laser active material, the doping concentration and the pump design. The disk is highly reflective coated on its back side for both the laser and the pump wavelengths and anti-reflectively coated on the front side for both wavelengths. This disk is mounted with its back side on a water-cooled heat sink. Due to this mounting and cooling technique, the temperature gradients inside the laser crystal are mainly coaxial to the disk axis and the laser beam axis. The temperature in the radial direction is nearly uniform within the homogeneously pumped central area of the disk. Therefore, these temperature gradients only slightly influence the laser beam propagation through the disk. All the thermal lens effects and the aspherical parts of the profile of the index of refraction are reduced by more than one order of magnitude compared with rod laser systems. The stress-induced birefringence is even further reduced and can be neglected for real laser systems. Additionally, due to the large surface-to-volume ratio, the heat dissipation from the disk into the heat sink is very efficient, thus allowing operation at extremely high volume power densities in the disk. Using multiple pump beam passes through the disk, results in a thinner disk and/or a lower doping concentration, thus reducing the thermal effects such as thermal lensing and stress in the disk. Another advantage is that the effective pump power density is increased so that on the one hand the demands to the power density of the pump diodes are reduced and on the other hand, quasi-three-level laser materials can also be used with this design. Quasi-three-level materials offer, on one hand, the possibility of building lasers of the highest efficiency. But on the other hand, they are hard to operate because they show a relatively high absorption of the laser-wavelength since the lower laser level is so close to the ground state that a considerable number of the laser-ions are in the lower laser level, when the laser is operated at room temperature. Therefore, it is necessary to pump the material with high pump power density in order to reach the threshold without increasing the temperature of the crystal too much. Using multiple pump beam passes through the crystal is therefore the key to achieve low threshold and high efficiency, because this helps to simultaneously reduce the thickness of the crystal and the doping concentration. This decoupling of laser and pump beam absorption is essential for the operation of quasi-three-level systems. The limit for the possible number of pump beam passes through the disk is given by the beam quality of the laser diodes which determines the beam diameter on the parabolic mirror and hence the number of positions on the mirror which can be used. The better the beam quality of the pump laser diodes, the higher the number of pump beam passes that are possible and the higher will be the total efficiency of the disk laser. When operating the disk in easy to scale the output power or energy just by increasing the pump spot diameter while keeping the pump power density constant. Also, there is no need to increase the brightness of the pump laser diodes. Besides quasi-three-level systems like Ytterbium and Thulium doped materials nearly all classical laser materials can be operated in the disk design, especially if the absorption of the pump radiation is quite high. This has been demonstrated by using $\mathrm{Nd}$ in YAG and other hosts.

Besides the outstanding properties of the disk laser design for CW-operation it is also well suited for P-P laser systems, especially if high average output power is required. Up to the present time, high average power P-P disk laser systems have been developed and demonstrated for the ns-pulse duration regime. That systems show an excellent beam quality and high efficiency. P-P operation could be achieved by inserting an AOM inside the 
laser resonator and not only. Using the concept of a master oscillator, followed by a regenerative amplifier allows for pulse amplification of ns-, ps- and fs pulses, the oscillator generates pulses with the desired properties (pulse duration, wavelength and repetition rate) which are amplified to the desired energy in the disk amplifier. The disk amplifier in this scheme is operating independently of the seed laser and is able to amplify any incoming pulse with the right wavelength and a pulse duration which is shorter than the round-trip time of the amplifier resonator. Simulations show that scaling of the output power of one single disk is only limited by amplified spontaneous emission (ASE) if the pump spot diameter becomes increasingly larger. Fortunately, the gain of low doped $\mathrm{Yb}$ : YAG is rather small so that ASE will occur only at very high pump power levels. For a 9 at $\%$ doped disk with a thickness of $200 \mu \mathrm{m}$ the power limit occurs at a pumping power beyond $50 \mathrm{~kW}$ so that much more than $20 \mathrm{~kW}$ laser power can be extracted in $\mathrm{CW}$-operation from one disk. This power level $(20 \mathrm{~kW})$ can be considerably further increased by increasing the pump spot diameter. The limitation set by ASE can then be overcome by using a disk with an undoped cap on top of the original disk, thus reducing the mean radial gain by the square of the ratio between the undoped and the doped material. The simulations also show that the laser power level for fundamental mode operation can be increased to nearly the same power level as for multi-mode operation. The reason for this behaviour is that the aspherical part of the residual thermal lens of the disk inside a top-hat pump profile is extremely low and independent of the pump spot diameter itself. The additional phase step at the edge of the pump profile is low and also nearly independent of the pump spot diameter. This phase step can be compensated for, by using simple adaptive optics. Scaling the pulse energy of single disk is more strongly limited by ASE than the power under CW-operation since the gain under low repetition rate P-P conditions is much higher compared with the CW- operation of a disk. Nevertheless, using an undoped cap on top of the disk and some other construction features (not presented here "know-how") will result in achievable energy levels far beyond $100 \mathrm{~J}$ from mono-module disk laser (MMDL) geometry.

The concept of TRUPF and BOEING is very much similar to this $\mathrm{CO}_{2}$ laser, which had been developed in 1970s by GPI RAS [31]. That approach was very much effective in understanding of many features of high power lasers development (high power optics, adaptive optics, CW plasma ball in air for new applications and so on). But this "Zig-Zag" approach for high power laser development is not effective, it is gone forever. The only way for multi-MW scale laser development is a MMDL with $\mathrm{D}>1 \mathrm{~cm}$ The MMDL is an innovative laser concept that allows one to build diode pumped solid state lasers with the highest average output powers, the highest efficiency and the best beam quality, simultaneously. Nearly all operational modes of solid state lasers such as CW, P-P operation can be built using our new design and having better properties than previous designs. Laser output average power of much more than existing level is possible due to our innovative efforts. The scaling laws for disk laser design according to our approach show that the average power limit is far beyond $1 \mathrm{MW}$ for a MMDL. Variation of the radiation temporal structure will help to overcome the very serious problems and to solve many new tasks:

1) To increase an effective energy deposition into a target;

2) To prevent the big loss of energy during propagation in air due to absorption saturation effect for high peak power of pulses;

3) To realize a much higher extraction of energy from a big volume of a laser active media.

\section{Conclusion}

Only the nearest future will show the details of physical-technical solution for the best laser which will be used effectively for many important challenges faced by science and technological advances of the future. However, today we can say that creation of high power class high frequency P-P lasers with a large cross section of the active medium, high compactness and low weight will open up a great prospect for their use in solving the problems of launching of small satellites by lasers, formation of super-long conducting channels in space for wireless power transmission (vacuum and atmosphere), cleaning of the near-Earth space from debris and many other special tasks of our days [32] [33].

\section{References}

[1] Apollonov, V.V., et al. (1991) High-Power Molecular Lasers Pumped by a Volume Self-Sustained Discharge. Journal of the Optical Society of America B, 8, 220-229. http://dx.doi.org/10.1364/JOSAB.8.000220

[2] Alexandrov, G.N., et al. (1980) Lasernyi perehvat molnii. Elektrichestvo, 12, 47.

[3] Kinoshita, F., Morooka, Y., Uchiumi, M., et al. (1997) Air Breakdown Produced by Pulsed $\mathrm{CO}_{2}$ Laser. Proceedings of 
XII International Conference on Gas Discharge and Their Applications, Greifswald, 69.

[4] Diels, J.-C. and Rudolph, W. (2006) Ultrashort Laser Pulse Phenomena: Fundamentals, Techniques, and Applications on Femtosecond Time Scale. Academic Press, Burlington.

[5] Kasparian, J., Rodriquez, M., M'ejean, G., et al. (2003) White-Light Filaments for Atmospheric Analysis. Science, 301, 61-64. http://dx.doi.org/10.1126/science.1085020

[6] Berge, L., Skupin, S., Nuter, R. and Wolf, J.-P. (2007) Ultrashort Filaments of Light in Weakly Ionized, Optically Transparent Media. Reports on Progress in Physics, 70, 1633. http://dx.doi.org/10.1088/0034-4885/70/10/R03

[7] Zvorykin, V.D., Levchenko, A.O. and Ustinovskii, N.N. (2011) Control of Extended High-Voltage Electric Discharges in Atmospheric Air by UV KrF-laser Radiation. Quantum Electronics, 41, 227. http://dx.doi.org/10.1070/QE2011v041n03ABEH014477

[8] Apollonov, V.V., Vasilyak, L.M., Kazantsev, S.Yu., et al. (2002) Electric-Discharge Guiding by a Continuous Spark by Focusing $\mathrm{CO}_{2}$-Laser Radiation with a Conic Mirror. Quantum Electronics, 32, 115. http://dx.doi.org/10.1070/QE2002v032n02ABEH002140

[9] Bazelyan, E.M. and Raizer, Yu.P. (2001) Physics of Lightning and Lightning Protection. Fizmatlit, Moscow.

[10] Apollonov, V.V. (2005) Feasibility Study of a $\mathrm{CO}_{2}$-Laser Based Lightning-Protection System Realization. Optical Engineering, 44, 1. http://dx.doi.org/10.1117/1.1829096

[11] Pyatnitskii, L.N. and Korobkin, V.V. (2000) Lasernyi proboi vozduha izlucheniem sfokusirovannym aksikonom. Trudy IOFAN, 57, 59.

[12] http://www.teramobile.org/publis.html.

[13] Bazelyan, E.M. and Raizer, Y.P. (1997) Spark Discharge. Izd-vo MFTI, Moscow.

[14] Aleksandrov, G.N. (1967) Lazernye metody controlya atmosfernogo electrichestva. Zhurnal Tekhnicheskoi Fiziki, 37, 288.

[15] Raizer, Y.P. (1974) Laser Spark and Propagation of Discharges. Nauka, Moscow.

[16] Asinovskii, E.I. and Vasilyak, L.M. (2000) Encyclopedia of Low-Temperature Plasma. Nauka, Moscow.

[17] Apollonov, V.V., Kiiko, V.V., Kislov, V.I., Suzdal'tsev, A.G. and Egorov, A.B. (2003) High-Frequency Repetitively Pulsed Operating Regime in High-Power Wide-Aperture Lasers. Quantum Electronics, 33, 753-757. http://dx.doi.org/10.1070/QE2003v033n09ABEH002496

[18] Apollonov, V.V. and Silnov, S.M. (2014) High Power P-P Lasers, NOVA.

[19] Apollonov, V.V. (2009) Proceedings of the X International Conference on Photonics and Optoelectronics, Wuhan, 4. RF Patent No. 2400005 May 20.

[20] Fuks, N.A. (1955) Mekhanika aerozolei, Mechanics of Aerosols. Izdatel'stvo AN SSSR, Moscow.

[21] Ageev, V.P., Barchukov, A.I., Bunkin, F.V., et al. (1977) Laser Air-Breathing Jet Engine. Kvantovaya Elektronika, 4, 2501. [Soviet Journal of Quantum Electronics, 7, 1430].

[22] Apollonov, V.V., Corkum, P.B. and Teylor, R.S. (1979) Selection of High-Power Nanosecond Pulses from LargeAperture $\mathrm{CO}_{2}$ Oscillators. Applied Physics Letters, 35, 147. http://dx.doi.org/10.1063/1.91054

[23] Apollonov, V.V., Kislov, V.I., Kijko, V.V. and Suzdal'tsev, A.G. (2003) High-Frequency Repetitively Pulsed Operation Regime in High-Power Wide-Aperture Lasers. $Q E, \mathbf{3 3}, 9$.

[24] Apollonov, V.V., Prokhorov, A.M. and Guenther, A.H. (2001) Power Optics, Problems, Developments, and Opportunities. Laser Physics, 11, 8.

[25] Apollonov, V.V. (2012) High Power Optics, Symposium HPLS@A-2012, Istanbul 10 September.

[26] Apollonov, V.V. (2012) New Operational Regime in High-Power Wide-Aperture Lasers. Proceedings of HPLA-2012, Santa Fe, (NM), USA, 35.

[27] Apollonov, V.V. (2012) High Power Lasers and Ecology of Atmosphere. Natural Science, 4, 713.

[28] Basov, N.G., Bogdankevich, O.V. and Grasiuk, A.Z. (1966) Semiconductor Lasers with Radiating Mirrors. IEEE Journal of QE, $2,9$.

[29] SPIE Events Europe (2012) TRUMPF's R@D.Internet.

[30] Giesen, A., Hugel, H., Voss, A., Wittig, K., Brauch, U. and Opower, H. (1994) Scalable Concept for Diode-Pumped High-Power Solid-State Lasers. Applied Physics B, 58, 365-372. http://dx.doi.org/10.1007/BF01081875

[31] Apollonov, V.V., Barchukov, A.I. and Prokhorov, A.M. (1971) Optical Surface Deformation under the Action of Laser Beam. JEEE Journal of QE, $\mathbf{6}, 10$.

[32] Apollonov, V.V. (2012) To the Space by Laser Light. Natural Science, 4, 719.

[33] Apollonov, V.V. (2014) High Power Optics. Springer, Berlin. 